\section{Dietmar Detering}

\section{Ökonomie der Medieninhalte}

Allokative Effizienz und soziale Chancengleichheit in den Neuen Medien

Münster/Hamburg/London: LIT Verlag, 2001. - $304 \mathrm{~S}$.

\section{ISBN 3-8258-5634-8}

Die hier besprochene Arbeit wurde im Sommersemester 1999 von der philosophischen Fakultät der Westfälischen Wilhelms-Universität Münster als Dissertation angenommen. Als Verlagsausgabe wurde sie, leicht gekürzt und ergänzt um aktuelle Entwicklungen, im Jahr 2001 im LIT Verlag veröffentlicht, der sie zu einem Preis von $30,90 €$ vertreibt. Sie kann aber auch, gegen Rechnung und versandkostenfrei, zum Preis von $23,00 €$ direkt beim Autor bestellt oder kostenlos aus dem Internet (www.medieninhalte.de) abgerufen werden.

In den drei ersten Hauptteilen seiner Arbeit versucht der Verfasser, wie im Klappentext angekündigt, ,erstmals eine umfassende Darstellung der wirtschaftlichen Eigenschaften urheberrechtlich geschützter Werke“ vorzunehmen. Hierzu beschreibt er (in Kapitel 2) die verschiedenen Funktionen von Medieninhalten, Motive für deren Nutzung, ihre ökonomischen Besonderheiten (wie Substituierbarkeiten, Nichtrivalität, Unteilbarkeiten, Externalitäten, Ausschließbarkeit u. a.), Formen der Produktion und des Zwischenhandels sowie mögliche Finanzierungsformen. Anschließend beschreibt er (in Kapitel 3), wie die Eigenschaften der "neuen“ (im Wesentlichen digitalen statt analogen und online abrufbaren statt trägergebundenen) Medien die Wirtschaftsstruktur, das Urheberrecht, die Finanzierungsformen und die Zugangschancen verändern.

Diese Teile referieren - im Allgemeinen korrekt und verständlich formuliert - den Wissenstand der neueren medienökonomischen Lehrbücher. Deutlich machen kann der Verfasser vor allem den für seine weitere Argumentation wichtigen Umstand, dass der bei einer fehlenden Preisdifferenzierung erforderliche Ausschluss von Konsumenten, deren Zahlungsbereitschaft unterhalb des (Einheits-)Preises, aber oberhalb der (für Medieninhalte geringen) Grenzkosten der Bereitstellung liegt, einen schwer wiegenden allokativen wie auch distributiven (d. h. hier: die sozialen Chancen beein- trächtigenden) Mangel darstellt. Auf der anderen Seite müssen die Ausführungen des Verfassers in diesen Teilen zwangsläufig knapp bleiben, so dass sie die in der Literatur geführten, zum Teil sehr differenzierten Auseinandersetzungen nicht vollständig und zum Teil auch nicht ausgewogen nachzeichnen. Das gilt etwa für die Darstellung und Kritik an der Finanzierung von Medieninhalten durch Werbung (Kapitel 2.5.2.) und durch eine Rundfunkgebühr (Kapitel 2.5.5.) oder für die veränderten Finanzierungsformen der digital und online abrufbaren neuen Medien, z. B. infolge abnehmender Transaktionskosten, zunehmender Bedeutung von Netzvorteilen oder intrinsischer Motive der Inhalteproduktion (Kapitel 3.4.).

Insgesamt vermittelt der Verfasser dem Leser in diesen Teilen seiner Arbeit aber durchaus das erforderliche Rüstzeug für seine darauf aufbauende „mikroökonomische Theorie der Medieninhalte". Diese entwickelt er, indem er zunächst (in Kapitel 4.1.) die allokativen Kriterien für die Beurteilung von Medieninhalten darlegt (,individuelle Wertezuweisung“, „Quantität der Inhalteangebote“, „Vielfalt des Inhalteangebots" und „Qualität der Inhalte“ und indem er (in Kapitel 4.2.) erklärt, wie Medieninhalte als öffentliche Güter (im Sinne nicht rivalisierender Güter) im LindahlGleichgewicht effizient und individuell gerecht bereitgestellt werden. Anknüpfend an die mit diesem Mechanismus verbundenen Probleme (fehlende Kenntnis unterschiedlicher Nachfrageintensitäten und opportunistisches Verhalten der Nachfrager), beschreibt Detering sodann verschiedene Formen der Preisdifferenzierung und deren wohlstandsfördernde und -schädliche Wirkungen, wobei er durch illustrative Beispiele deutlich macht, dass es sich hierbei keinesfalls um abstrakte Modellüberlegungen handelt, sondern um bei Medieninhalten durchaus gängige Geschäftspraktiken (denen Detering sich bei der Vermarktung seiner Arbeit im Übrigen ja auch selbst bedient).

In den Kapiteln 4.3. und 4.4. behandelt der Verfasser sodann eine Vielzahl ökonomischer Modelle der privaten Bereitstellung öffentlicher Güter im Wettbewerb. Die Bereitstellung homogener öffentlicher Güter mit Preisdifferenzierung beschreibt er anhand von Modellen von Thompson, Demsetz und Oakland; für die Bereitstellung differenzierter öffentlicher Güter berücksichtigt er die Theorie des monopolistischen Wettbewerbs, das Steiner-Rothen- 
berg-Beebe-Modell, das Spence-Owen-Modell sowie Modelle des monopolistischen Wettbewerbs im so genannten Produktraum. Obwohl sich die Erkenntnisinteressen, die betrachteten Variablen und auch die Terminologien dieser Modelle zum Teil beträchtlich voneinander unterscheiden, gelingt es Detering, die Modelle miteinander zu vergleichen und daraus Schlussfolgerungen für die zuvor benannten Kriterien allokativer Leistungsfähigkeit einer privaten Bereitstellung öffentlicher Güter zu ziehen. Unter Rückgriff auf die zuvor dargestellten Besonderheiten der Inhalte von (neuen) Medien kann er auch belegen, dass die mit diesen Modellen ableitbaren Ergebnisse nur mit zum Teil massiven Einschränkungen oder Erweiterungen auf den Markt für Medieninhalte übertragen werden können. Er unterstreicht damit zum einen, dass die (etwa von der so genannten TV-Economics-Schule) entwickelten Bereitstellungsmodelle ihre Stringenz, Eindeutigkeit und didaktische Überzeugungskraft in dem Umfang verlieren, in dem sie an die Realität angepasst werden; zum anderen macht er deutlich, warum die markt- und wettbewerbstheoretischen Empfehlungen, die für die „klassischen“ Güter der mikroökomischen Lehrbücher entwickelt worden sind, für Medienmärkte wenig taugen.

Detering knüpft hieran aber nicht die Forderung nach einer stärkeren Regulierung einer marktlichen Bereitstellung oder gar nach öffentlicher Bereitstellung von Medieninhalten an, sondern schlägt (in Kapitel 5) eine Verbesserung des privaten Bereitstellungsmechanismus vor. Dabei knüpft er an den Bedingungen für die Zuweisung perfekt differenzierender Lindahl-Preise an, die darin bestehen, dass erstens jedem Nachfrager die einzelnen Medieninhalte zu den Grenzkosten ihrer Bereitstellung zugänglich sind und zweitens für den $\mathrm{Zu}-$ gang zu einem Medieninhalt nur der Preis entrichtet werden muss, der der allein auf diesen Inhalt zurückzuführenden Erhöhung des gesamten (individuell bewerteten) Nettonutzens aller Medieninhalte entspricht. Aufbauend auf dem von Vickrey, Clarke, Croves und anderen Autoren entwickelten ,demand-revealing process “ für öffentliche Güter schlägt Detering mit dem "Collective Pricing of Differentiated Goods", CPDG, (in Kap. 5.1.) ein privates Bereitstellungsverfahren für Medieninhalte vor, das diese Bedingungen erfüllt, indem es die Nachfrager in ihrem eigenen Interesse (der Ma- ximierung ihrer Konsumentenrenten) zwingt, ihre wahren Präferenzen offen zu legen und damit zugleich die Voraussetzungen für eine gesellschaftlich optimale Allokation zu schaffen.

In der praktischen Anwendung wirft dieses Verfahren eine Vielzahl von Fragen und Problemen auf (hohe Transaktionskosten, mangelnde Homogenität und Stabilität von Gemeinschaften mit gleicher Zahlungsbereitschaft, mangelnde Akzeptanz des Verfahrens durch die Nachfrager u. a.). Diese werden von Detering, zum Teil unter Rückgriff auf Arbeiten der Public Choice Schule (5.2), zum Teil unter Einbezug neuerer Überlegungen zur Bildung "autostabiler virtueller Gemeinschaften“ (Kap. 5.3.) und zum Einsatz von Auktionen als alternativen Verfahren der Preisdifferenzierung (Kap. 5.5.) diskutiert. Wenngleich es dem Verfasser damit nicht gelingt, die gegenüber "complicated alternatives to majority rules" (z. B. von Mueller in seiner Public Choice II, 1989) vorgebrachten Bedenken vollständig zu zerstreuen, ist die Arbeit von Detering auch in diesem Teil eine lehrreiche, originelle und anregende Arbeit, deren Lektüre uneingeschränkt empfohlen werden kann.

Zu knapp fällt allerdings die Darstellung und Bewertung der mit dem Verfahren verbundenen distributiven Konsequenzen aus; anders als es im Untertitel ausgedrückt wird, konzentriert sich die Arbeit auf die allokative Effizienz der Bereitstellung von Medieninhalten. Das ist bedauerlich, da man sich von Detering eine ähnlich ambitionierte und kenntnisreiche Behandlung der mit den verschiedenen Bereitstellungsverfahren von Medieninhalten verbundenen distributiven Wirkungen wünschen würde. Die Chancen hierfür scheinen aber schlecht zu stehen, da der Autor, wie man auf seinen Internetseiten nachlesen kann, mittlerweile ein von ihm mitgegründetes und in New York ansässiges Unternehmen zur Direktvermarktung von Events über das Internet betreibt.

Da dieses Unternehmen zum Zwecke der zielgenauen Adressierung von Event-Einladungen detaillierte Daten über die soziodemographischen Merkmale und die beruflichen und privaten Interessen und Aktivitäten derjenigen sammelt, die sich einladen lassen wollen, könnte uns der Verfasser im Übrigen auch bezüglich unserer Bedenken gegen die Praktikabilität seines CPDG-Verfahrens eines Besseren belehren, wenn er mit diesen Daten, 
abweichend von ihrem ursprünglichen Verwendungszweck, ,autostabile virtuelle $\mathrm{Ge}$ meinschaften " für ein "Collective Pricing of Differentiated Goods" bilden würde. Ein USPatent dafür hat Herr Detering jedenfalls schon mal beantragt (siehe www.medieninhalte.de/patent/collectivepricing.htm). Geben Sie Ihre persönlichen Daten bitte ein unter www.eventme.com ...

Manfred Kops

Tilman Sutter / Michael Charlton (Hrsg.)

Massenkommunikation, Interaktion und soziales Handeln

Wiesbaden: Westdeutscher Verlag, 2001. 274 S.

ISBN 3-531-13629-1

Der vorliegende Band dokumentiert eine Tagung an der Universität Freiburg im Herbst 1999. Die Fragestellungen, welche die Tagung überspannten, waren, in welchem Verhältnis Interaktion in der Alltagskommunikation und Massenkommunikation zueinander stehen, in wiefern erstere ein Modell für letztere sein kann (oder soll) und wie eine Beziehung zwischen subjektiven Aneignungsprozessen massenmedialer Inhalte, ihrer kommunikativen Verarbeitung in der Interaktion und den Prozessen der Massenkommunikation allgemein hergestellt werden kann.

In der Einleitung konstatiert Tilmann Sutter nicht nur einen „Kooperationsbedarf zwischen system-, handlungs- und subjekttheoretischen Untersuchungsperspektiven in der Medienforschung" (S. 10), sondern vielmehr die Notwendigkeit, ihre „Gegenstellung “ zu überwinden (S. 9). Man kann also - etwas überspitzt -das Bemühen um eine einheitliche Feldtheorie der Rezeptionsforschung als Anliegen dieses Bandes diagnostizieren.

Das Buch ist in drei Abschnitte untergliedert, die in ihrer Anordnung den bekannten Verlauf von Tagungen unterstellen: Sutter und Charlton stellen zur „Eröffnung der Debatte“ die Beziehungen und Unterschiede zwischen Massenkommunikation, individueller Rezeption und sozialer Anschlusskommunikation bzw. sozialer kommunikativer Aneignung jeweils aus system- und handlungstheoretischer Perspektive dar. Der zweite Teil des Bandes versammelt „theoretische Positionen“ zu Phä- nomenen wie Anonymität (Esposito) und Intersubjektivitätsproduktion (Schneider), den interaktionstheoretischen Aspekten bei Adorno (Müller-Doohm), dem Zusammenhang von Medienprodukt und seinem sozialen Gebrauch (Keppler) und methodischen Implikationen einer handlungstheoretischen Kommunikationsforschung (Renckstorf/Wester). Im dritten Teil werden schließlich „Reflexionen anhand empirischer Fallanalysen“ angestellt: Hausendorf befasst sich mit der Funktion der Publikumsansprache, Holly und Habscheid interpretieren Gattungen als soziale Muster der Fernsehkommunikation und Ayaß weist exemplarisch die Inszenierung von Spontaneität nach.

In seinem Beitrag „Sinnstrukturen der Medienkommunikation“" zur „Eröffnung der Debatte“ geht Sutter davon aus, dass die verschiedenen Forschungsrichtungen zu einer „umfassenden Konzeption von Medienkommunikation zusammengeschlossen werden " können. Sein Versuch, Systemtheorie und verstehende Ansätze zu integrieren, scheitert jedoch prinzipiell daran, dass er nur Erstere detailliert expliziert und Letztere als einheitliches Ganzes behandelt - was sie selbstverständlich nicht sind, und was in den nachfolgenden Beiträgen auch deutlich wird. Sutter steuert argumentativ auch gar nicht auf diese Integration zu, sondern widmet sich der Frage, wie die drei Dimensionen der Medienkommunikation (Massen-, Anschlusskommunikation und Rezeption) selbst nun systemtheoretisch zusammengeführt werden können. Mit dem Begriff der strukturellen Kopplung gelingt es innerhalb der Logik dieses Ansatzes durchaus, die jeweiligen Eigengesetzlichkeiten dieser drei Dimensionen zu bewahren und gleichzeitig den Zusammenhang herauszuarbeiten, der zwischen diesen aufeinander prinzipiell verwiesenen Kommunikationsprozessen besteht. Auch wenn dieser Zusammenhang mittels einer Metapher beschrieben wird, die so gar nicht zur rationalisierenden Sprache der Luhmann'schen Systemtheorie passen will: Sutter spricht von einem "doppelten Spiegel“ (36), der zwischen den Medienakteuren und dem Publikum steht. Da fragt man sich schon, aus was dieser Spiegel denn bestehen könnte und wie er dorthin kam. Dennoch ist dieser Begriff tatsächlich insofern bereichernd, als er in seiner Einprägsamkeit die Leserin während der weiteren Lektüre begleitet und zum Beispiel bei den verschiedenen Ausführungen anderer 